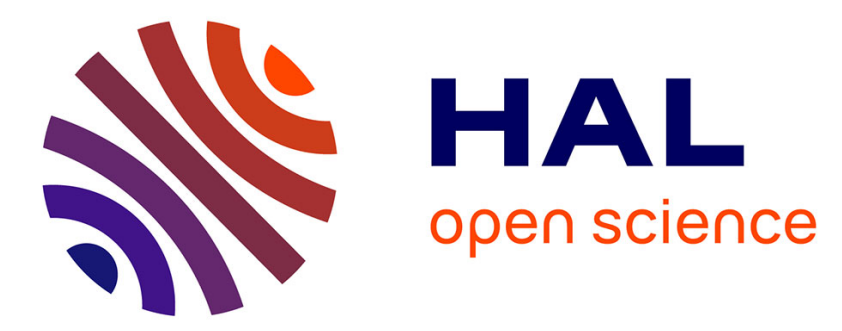

\title{
Saint Jean le Baptiste dans les chapelles peintes du palais des papes d'Avignon et de la chartreuse de Villeneuve (1347 et 1355)
}

Véronique Rouchon Mouilleron

\section{- To cite this version:}

Véronique Rouchon Mouilleron. Saint Jean le Baptiste dans les chapelles peintes du palais des papes d'Avignon et de la chartreuse de Villeneuve (1347 et 1355). L'Eglise et la vie religieuse, des pays bourguignons à l'ancien royaume d'Arles (XIVe-XVe siècle), CENTRE EUROPEEN D'ETUDES BOURGUIGNONNES (XIVe-XVIe s.), 2009, AVIGNON, France. halshs-01967163

\section{HAL Id: halshs-01967163 \\ https://shs.hal.science/halshs-01967163}

Submitted on 30 Dec 2018

HAL is a multi-disciplinary open access archive for the deposit and dissemination of scientific research documents, whether they are published or not. The documents may come from teaching and research institutions in France or abroad, or from public or private research centers.
L'archive ouverte pluridisciplinaire HAL, est destinée au dépôt et à la diffusion de documents scientifiques de niveau recherche, publiés ou non, émanant des établissements d'enseignement et de recherche français ou étrangers, des laboratoires publics ou privés. 


\section{VÉRONIQUE ROUCHON MOUILLERON}

Université Lumière Lyon II - CIHAM-UMR 5648

\section{SAINT JEAN LE BAPTISTE DANS LES CHAPELLES PEINTES DU PALAIS DES PAPES D'AVIGNON ET DE LA CHARTREUSE DE VILLENEUVE (1347 ET 1355) *}

Rapportés à l'omniprésence de la figure médiévale de Jean-Baptiste dans le répertoire visuel de la péninsule italienne, et à son intégration dans l'histoire politique et religieuse de 1'Italie des communes ${ }^{1}$, les deux cycles picturaux qui lui sont consacrés sur les murs du palais des papes d'Avignon et ceux de la chartreuse de Villeneuve invitent à tenter une relecture ponctuelle. Une telle approche conjuguée paraît légitime, puisque ces peintures sont, en ces deux sites, attribuées à un artiste également originaire d'Italie, le célèbre Matteo Giovannetti, parce qu'elles sont l'œuvre d'un seul et même peintre, qui les a exécutées à moins de dix ans de distance, au milieu du XIVe siècle, à quelques kilomètres l'une de l'autre, et pour répondre à deux commanditaires également papes, Clément VI et Innocent VI.

Si l'ample question de la migration des images embrasse le plus souvent des œuvres géographiquement, chronologiquement et historiquement éloignées, d'une rive à l'autre de la Méditerranée, entre Orient et Occident, centre et périphérie ou

* Je tiens à remercier pour leur concours Mme Sophie Biass-Fabiani, conservatrice du palais des papes, et M. François de Banes Gardonne, directeur de la chartreuse de Villeneuve-lèsAvignon.

1 Qu'il nous soit permis de renvoyer à notre article Entre Orient et Occident: l'image de saint Jean du XIe au XIVe siècle, dans Revue de l'art, $\mathrm{n}^{\circ} 158$ / 2007-4, Art médiéval de la Méditerranée, pp. 35-45 ; ainsi qu'à la notice Baptistère dans Dictionnaire d'histoire de l'art du Moyen Age occidental, sous la dir. de P. Charron - J.M. Gullouët, Paris, 2009, pp. 112-114. 
entre Antiquité tardive et Moyen Age central ${ }^{2}$, il est toutefois légitime d'envisager ici ces notions de transfert et d'adaptation iconographiques, mais en les transposant sur une faible échelle, comme une sorte de cas d'école en réduction : sur une petite décennie, d'une rive à l'autre d'un même fleuve (mais d'un Rhône autant trait d'union que frontière ${ }^{3}$ ), d'un chantier pontifical à un autre (mais selon les destinations divergentes d'un palais pour la curie et d'un monastère pour des chartreux).

L'analyse détaillée de ces images du Précurseur, que nous avons menée dans les chapelles avignonnaise et villeneuvoise, montre qu'au-delà des similitudes évidentes, les deux ensembles peints ici et là sont loin de fonctionner comme le décalque l'un de l'autre. Dans son ouvrage fondateur consacré à Matteo Giovannetti et à la peinture en Provence au XIVe siècle, initialement paru en 1962, et toujours invoqué jusqu'à aujourd'hui, Enrico Castelnuovo n'a pas manqué de mener un travail comparatiste entre le palais et la chartreuse, mais ses réflexions portent principalement sur les aspects stylistiques de ces peintures, avec, par exemple, des remarques centrées sur les raffinements progressifs du traitement de l'espace, dans l'une et l'autre chapelle, sur les trouvailles en matière de perspective tant atmosphérique qu'architecturale ${ }^{4}$. Vingt ans plus tard, dans l'ouvrage qu'ils consacrent à l'école d'Avignon ${ }^{5}$, Michel Laclotte et Dominique Thiébaut, en 1983, précisent la description de chacune de ces images, en identifiant les personnages, en reprenant la transcription de l'ensemble (ou presque) des inscriptions, en insistant encore sur les remarquables effets d'illusionnisme qui se dégagent de chaque scène $^{6}$. L'enquête conduite par Christian Heck sur la représentation conjointe du Baptiste et de Jean l'Evangéliste dans l'imagerie médiévale lui a permis d'exposer, en 1978, une première approche iconographique globale des peintures de la

2 Dans une bibliographie internationale fournie, nous retiendrons particulièrement les nombreuses contributions de Hans Belting et de Herbert L. KessLer, ainsi que les synthèses récentes proposées par D. Russo, Byzance et l'art médiéval: la question de l'intermédiaire italien, dans Revue de l'art, $\mathrm{n}^{\circ} 158$ / 2007-4, Art médiéval de la Méditerranée, pp. 5-12; et M. BAcci, L'arte : circolazione di modelli e interazioni culturali, dans Storia d'Europa e del Mediterraneo, éd. A. Barbero, sez. IV. Il Medioevo (secoli V-XV), vol. IX. Strutture, preminenze, lessici comuni, sous la dir. de S. CAROCCI, Rome, 2007, pp. 581-632.

3 J. Rossiaud, Le Rhône au Moyen Age, Paris, 2007.

4 E. Castelnuovo, Un pittore italiano alla corte di Avignone, Matteo Giovannetti e la pittura in Provenza nel secolo XIV, Turin, 1962, rééd. augmentée 1991, trad. française, Paris, 1996. Dans la réédition de 1991, et la traduction de 1996, voir en particulier les chapitres 8 et 10 , pp. 103-121 et 134-152 (1991), pp. 115-130 et 143-157 (1996) .

5 La définition d'une «école d'Avignon» a été proposée dès 1960 par M. LACLotte, L'école d'Avignon : la peinture en Provence aux XIVe et XVe siècles, Paris, 1960.

6 M. Laclotte et D. ThíBaut, L'école d'Avignon, Paris, 1983, pp. 35-44, 169-178 et 184192. La réédition de l'ouvrage, toujours chez Flammarion, en 1985, n'en propose qu'une forme abrégée. La transcription des inscriptions sera complétée avec celle donnée par L.-H. LABANDE, Le palais des papes et les monuments d'Avignon au XIVe siècle, Marseille, 1925, vol. 2, pp. 53-60 et pp. 172-176. Les transcriptions que nous proposons ici ont été vérifiées et légèrement remaniées quand nécessaire. 
chapelle Saint-Jean, où figurent les deux saints homonymes. Sa lecture, attentive à l'implantation spatiale des scènes par rapport à l'autel et au célébrant, s'oriente plus précisément vers les occurrences visuelles du rapprochement des deux Jean, qu'il rattache au modèle romain de la basilique du Latran ${ }^{7}$.

Ainsi, pour les vies du Prodrome peintes à Avignon et à Villeneuve, l'examen iconographique des thématiques picturales restait encore à peu près intégralement à fournir, autour du nombre de scènes déployées dans les deux cas, de leur positionnement respectif, mais aussi autour de la tradition visuelle dans laquelle chaque image doit être replacée, et qui inscrit les choix picturaux dans des projets spirituels ou culturels qu' il faut moduler. Notre étude portera done sur ces variantes, discrètes ou déclarées, qui donnent à la figure de Jean-Baptiste de réelles inflexions, entre curie et chartreuse.

C'est donc au prêtre, originaire de Viterbe, Matteo Giovannetti, et à l'atelier qu'il dirige, que sont attribuées les peintures qui nous occupent. Si le nom de Giovannetti est attesté entre 1322 et 1369 , les mentions les plus anciennes concernent ses seules charges ecclésiastiques. Comme peintre, il est signalé à partir de 1343, à Avignon et dans le royaume de France, jusqu'en avril 1367, puis au Vatican où il suit Urbain en 1368, au service des trois papes Clément VI, Innocent VI, puis Urbain V. A la cour avignonnaise, où il est qualifié successivement de magister, puis de pictor pape (en 1346), il n'est pas seulement à la tête d'un important atelier des peintres, mais en position de surintendant à la décoration des chantiers pontificaux ${ }^{8}$.

Au palais, dans la chapelle Saint-Jean, Giovannetti travaille entre 1346 et 1348 et sans doute plus précisément en $1347^{\circ}$. La pièce, attenante à la salle du consistoire où se réunissaient cardinaux et pape, s'ouvre sur son flanc Est, et fonctionne donc comme chapelle du consistoire. Mesurant environ $30 \mathrm{~m}^{2}$, de forme presque carrée, elle est percée de trois baies vitrées sur ses côtés Est, Nord et Sud et voûtée d'une ogive quadripartite. Les peintures $s^{\prime} y$ déploient sur les quatre murs et les quatre voûtains. Les histoires peintes des deux Jean, le Baptiste et l'Evangéliste, se partagent l'espace pictural, équitablement en termes de quantité, deux pans de mur et deux voûtains chacun, mais non en termes de positionnement, dans la mesure où

7 C. HEck, La chapelle du consistoire et les crucifixions dans la peinture murale avignonnaise du XIVe siècle: renouvellement d'un thème d'origine romaine au service de l'affirmation de la légitimité pontificale, dans Genèse et débuts du Grand Schisme d'Occident, Actes du 586e colloque international du CNRS (Avignon, 25-28 septembre 1978), Paris, 1980, pp. 431-443.

8 Pour une biographie détaillée, nous renvoyons aux travaux d'Enrico Castelnuovo, qui signale l'ensemble des sources utiles, éditées ou non. Une synthèse chez W. AngeleLl, Giovannetti Matteo, dans Dizionario Biografico degli Italiani, vol. 55, 2000, pp. 506-510. Des éléments de réflexion sur la place de Giovannetti dans le chantier pontifical chez E. ANHEIM, L'artiste et l'office. Financement et statut des producteurs culturels à la cour des papes au XIVe siècle, dans Offices, écrit et papauté (XIIT-XVIIe siècle), sous la dir. d'A. Jamme et O. Poncet, Rome-Paris, 2007 (Collection de l'Ecole Française de Rome 386), pp. 393-406.

9 E. Castelnuovo, op. cit., pp. 103-104 (1991), pp. 115-116(1996). 
l'histoire du Précurseur peut être embrassée du regard dès l'entrée, parce qu'elle occupe le mur qui fait face à la porte, à l'aplomb de l'autel, et sur le pan gauche adjacent, alors que l'histoire de l'Evangéliste se déroule sur la paroi de droite et le mur de revers, au-dessus de l'entrée ${ }^{10}$.

La vie du Baptiste se développe sur deux registres narratifs ${ }^{11}$. Elle débute en face, au mur Est, au registre supérieur, à droite et à gauche de la baie en lancette avec l'Annonce à Zacharie puis la Naissance avec Imposition du nom de Jean ; elle se poursuit en bas, par plusieurs épisodes unifiés dans un même paysage, où des hommes se dénudent pour être baptisés par Jean, tandis que, de part et d'autre de l'embrasure, celui-ci indique le Christ de la main (Ecce Agnus Dei). Le récit continue sur le côté gauche, au Nord, au registre supérieur, à nouveau avec une grande scène regroupant plusieurs tableaux : à droite, le moment des questions des prêtres et lévites au Prodrome l'interrogeant sur son identité et la source de son autorité, puis à gauche le Baptême du Christ suivi, au centre, de la théophanie trinitaire du Christ en union avec le Père et la colombe de l'Esprit (Mt 3, 13-17 ; Mc 1, 9-11; Lc 3, 21-22). Il s'achève en bas, sur la droite, avec la décapitation du martyr, puis à gauche, avec le Festin d'Hérode où est apportée la tête de Jean, tandis que l'embrasure gauche montre Salomé offrant le chef décollé à Hérodiade.

L'ordre et l'agencement des scènes méritent cependant quelques remarques. La lecture de l'histoire de droite à gauche et de haut en bas, que nous reprenons ici, ne peut, en effet, être invoquée de façon rigide. Elle fonctionne strictement pour les deux premiers tableaux isolés sur le mur de face (Annonce et Naissance), ainsi que pour la Décollation sur le pan septentrional. Mais après le martyre, en accolant les deux présentations du chef de Jean à la manière des volets d'un dyptique, qui forment un pendant entre la salle ouverte du Festin d'Hérode et la pièce close où Salomé rejoint sa mère, le dispositif pictural brouille toute précision chronologique sur la succession de ces moments posthumes (fig. 1).

10 C. Heck, op. cit., pp. 434-435. L'auteur choisit d'insister sur l'équilibre de l'espace mural octroyé à l'un et l'autre saint. Toutefois la disposition privilégiée du cycle du Baptiste invite à avancer l'hypothèse d'une prééminence visuelle de sa figure, d'autant que la question de la prédominance de l'un des deux saints Jean donne lieu à une vraie querelle de dévotion, ainsi que Jacques de Voragine le rapporte pour la fin du XIIIe siècle. Voir JACQues de Voragine, La légende dorée, éd. A. Boureau avec la collaboration de M. Goullet, P. Collomb, L. Moulinier et S. Mul A, Paris, 2004 (Collection Bibliothèque de la Pléiade 504), p. 441 et n. 24, p. 1264.

11 Dans l'impossibilité matérielle de publier ici l'ensemble du cycle johannique, nous renvoyons aux clichés mis à disposition sur le site du Ministère de la Culture et de la Communication consacré aux chapelles du palais des papes, élaboré en 1999 sous la direction scientifique de M.-C. LÉonelli et S. Kovalevsky [En ligne] URL : http://www.culture.gouv.fr/culture/palaisdes-papes/fr/acceuil.html [adresse valide en novembre 2009 - il faut bien lire «acceuil»...]. 


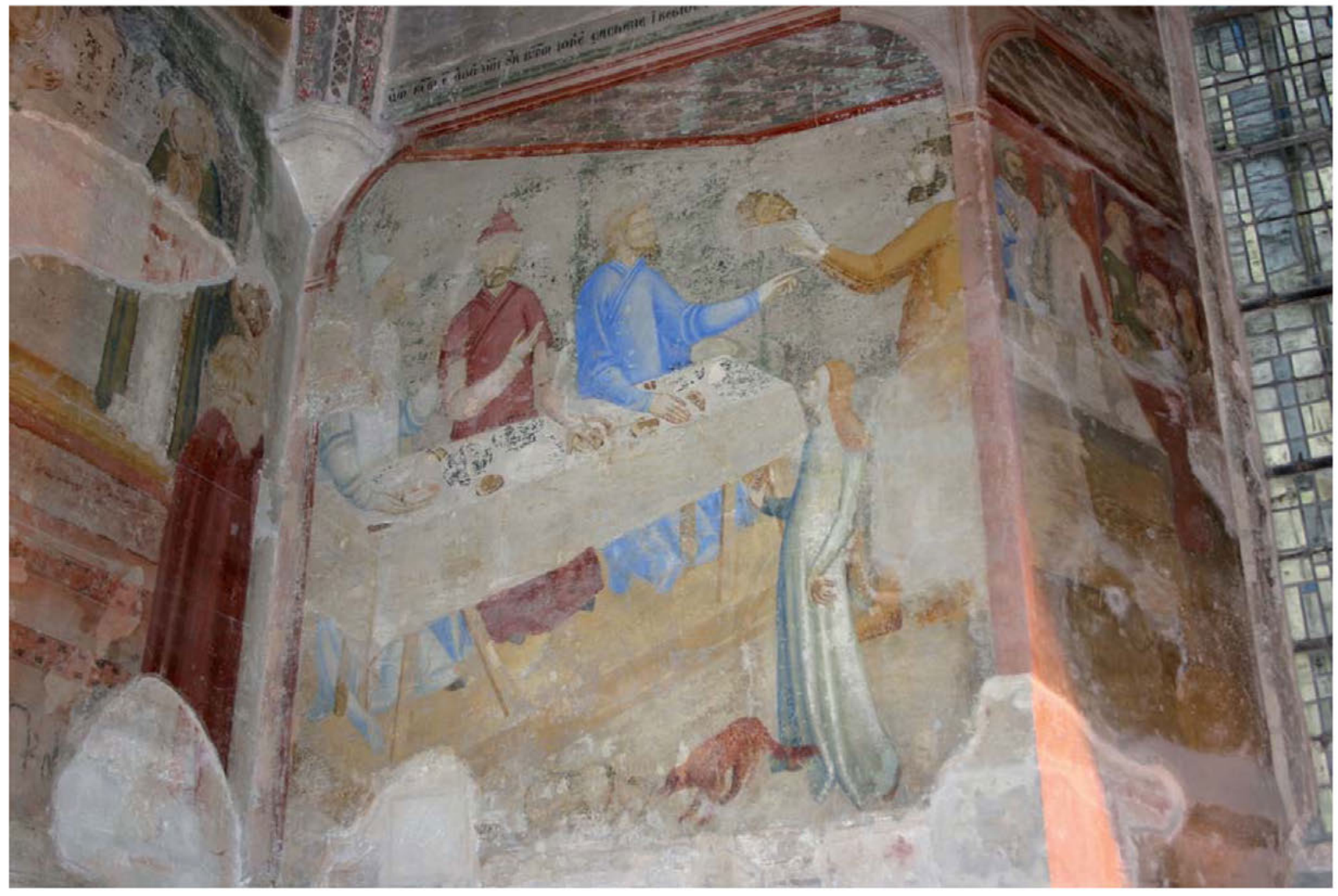

Figure 1 : Avignon, palais des papes, chapelle du consistoire, mur nord, registre bas, cycle de Jean le Baptiste : Remise du chef de Jean décollé au cours du Festin d'Hérode (pan gauche) et par Salomé à sa mère Hérodiade (embrasure de la baie) 


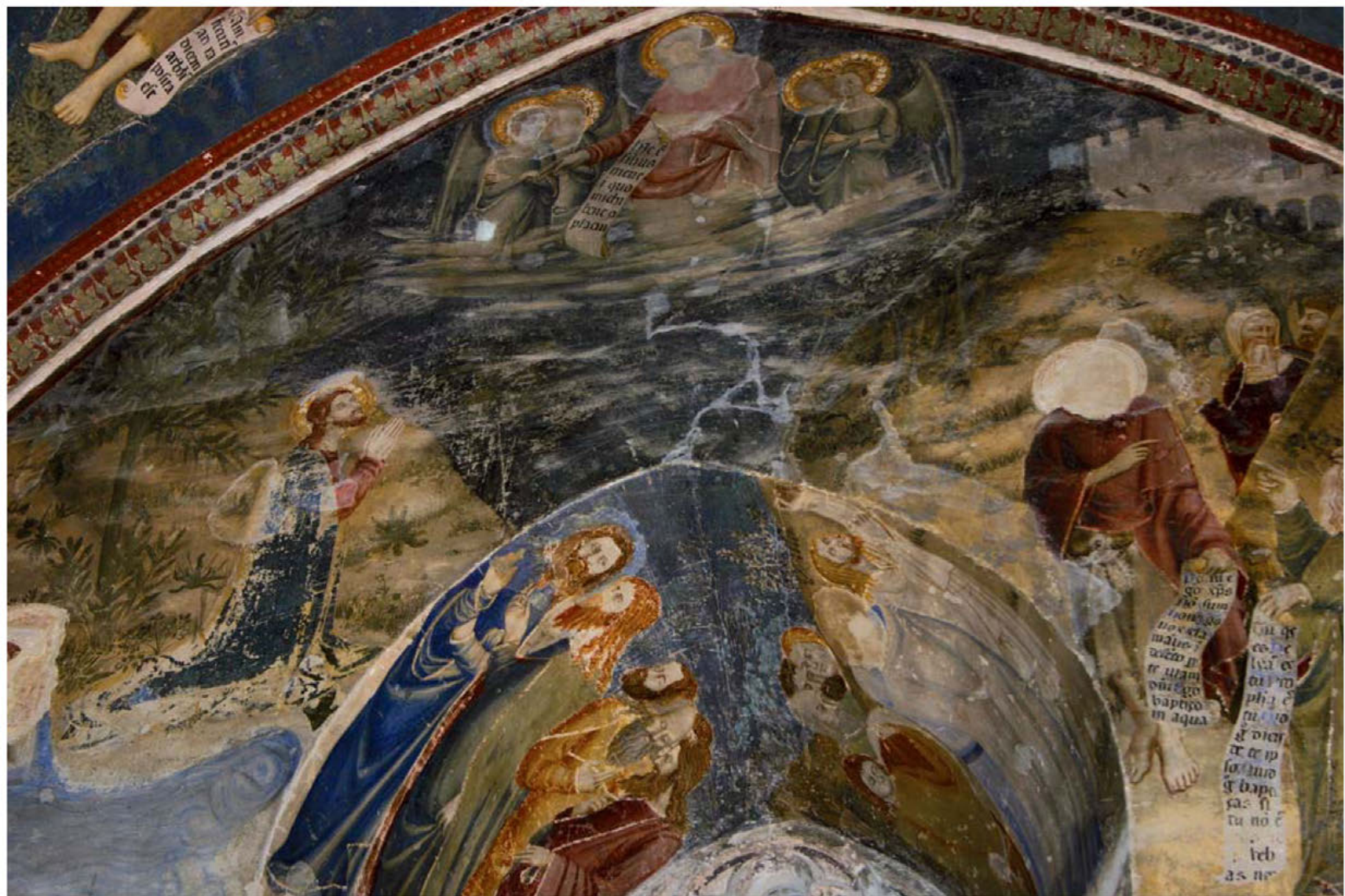

Figure 2 : Avignon, palais des papes, chapelle du consistoire, mur nord, cycle de Jean le Baptiste: Paysage unifié avec Jean interrogé par les pharisiens (à droite), Jean baptisant le Christ (à gauche) et Théophanie baptismale (au centre). 
De même, l'emplacement respectif des deux grandes scènes paysagées, au mur Est registre bas et au mur Nord registre haut, fait problème. Si, dans une approche textuelle, on regarde à la lettre le premier chapitre du quatrième évangile, la séquence est la suivante, décrite sur trois jours : $1^{\circ}$ questions des pharisiens au Baptiste (Jn 1, v. 19-28) ; $2^{\circ}$ «le lendemain », témoignage du Précurseur dans lequel il prononce le premier Ecce Agnus (v. 29-31), et décrit la descente de l'Esprit sur le Christ (v. 32-33), mais, rappelons-le, sans référence explicite au baptême ; puis $3^{\circ}$ à nouveau «le lendemain», deuxième Ecce Agnus (v. 35-36) suivi de la sequela des premiers disciples (v. 37). Encore une fois, la chronologie du texte et l'implantation de la peinture se télescopent, au profit de fondus enchaînés d'un registre à l'autre, d'un pan à l'autre ${ }^{12}$. Ces cas de figure invitent au dépassement du simple suivi littéral du récit au profit d'une lecture intériorisée, qui rassemble tous les moments du témoignage johannique non à la façon d'étapes progressives d'une démonstration, mais comme les équivalents d'une seule et même évidence. L'organisation des scènes sous-tend l'embrassement d'un regard panoramique. Cette idée se dégage particulièrement de l'ample paysage supérieur du mur gauche où Jean décline son identité auprès des pharisiens, où le baptême du Christ lui confère son identité de baptiseur, où l'apparition du Père identifie Jésus comme son Fils (fig. 2). Ainsi cette grande scène triple offre, sous trois facettes simultanées et indissociables, la réponse complète aux questions identitaires posées au saint.

Si l'on aborde le cas de Villeneuve, les conditions du chantier de la chartreuse sont moins claires qu'au palais. Résumons-les cependant : le pape Innocent VI décide d'employer le terrain et les bâtiments de son ancien palais cardinalice à la création d'une maison cartusienne. La bulle de fondation est promulguée le 2 juin 1356 et le monastère mis sous le vocable de Jean-Baptiste ${ }^{13}$. Les peintures de Giovannetti ornent la chapelle qui prolonge l'ancien grand tinel du cardinal, et qui deviendra le réfectoire des moines. Toutefois, les travaux d'E. Castelnuovo ont avancé, sur la base de suggestions archivistiques, la date de 1355 pour l'exécution de ces peintures, en proposant deux hypothèses : soit Innocent VI avait commandé une réplique du cycle décorant la chapelle consistoriale d'Avignon, qui a pu être adaptée ensuite à la nouvelle fondation, soit son projet de chartreuse l'occupait déjà à cette date, et le choix iconographique du Baptiste en dérive ${ }^{14}$. Il me semble même que les deux lectures ont tout lieu d'être posées ensemble : à la fois, redoubler la chapelle du consistoire d'Avignon, d'autant que des consistoires cardinalices se tiennent dans le grand tinel villeneuvois avant $1356^{15}$; et dans le même temps,

12 A noter que le site en ligne (URL cit.) propose de lire à la suite les deux registres supérieurs des deux pans, de droite à gauche, puis d'enchaîner les deux registres inférieurs.

13 J. Girard, Evocation du vieil Avignon, Paris, 1958, pp. 370-379; A. GRARD, Implantation cartusienne et architecture gothique autour d'Avignon, dans La naissance des chartreuses, sous la dir. de B. Bligny et G. ChaIx, Actes du VIe colloque international d'histoire et de spiritualité cartusiennes (Grenoble 12-15 sept. 1984), Grenoble, 1986, pp. 119-160.

14 E. Castelnuovo, op. cit., pp. 134-137 (1991), pp. 143-146 (1996).

15 Dans le document signalé par E. Castel nuovo, op. cit., n. 6, p. 136 (1991) et p. 145 (1996), le tinel est précisément désigné sous l'expression "pro consistorio Villenove». 
anticiper la future destination cartusienne de cette chapelle. Dans tous les cas, le cycle d'Avignon apparaît comme un argument ou un modèle prégnant, dont on ne peut faire l'économie dans la décennie qui suit son exécution. Par ce biais, Innocent VI pose les jalons de l'aménagement de ce qu'on peut appeler une chartreuse pontificale, ce que démontrera en termes explicites, à partir de 1360 , l'implantation de son tombeau dans l'église monastique - quand les Coutumes de Guigues réservent une sépulture dans le couvent aux seuls moines de l'ordre et que, même au XIVe siècle, ce choix s'avère rare dans la pratique cartusienne ${ }^{16}$.

La chapelle de Villeneuve-lès-Avignon correspond à la terminaison polygonale de la salle rectangulaire du tinel — ce qui n'est plus perceptible depuis que l'en sépare un mur plein percé d'une simple porte basse. De proportion barlongue, elle se compose de deux murs parallèles rectilignes, et de trois pans sur plan tournant, le tout voûté d'une ogive sexpartite. Le cycle du Baptiste occupe les deux pans droits, en face à face ${ }^{17}$. Sur le mur de droite, percé d'une baie, se lisent, disposés sur deux registres superposés : en haut, à gauche, l'Annonce à Zacharie, à droite la Visitation; en bas, à gauche, la Naissance du Précurseur, à droite, sa Circoncision et l'Imposition de son nom. Sur le mur d'en face, la lancette double à meneau a été bouchée et utilisée pour le décor pictural (fig. 3). Les scènes de gauche sont endommagées et ne sont identifiables que par leurs inscriptions ou leur contexte. En haut Jean en prison envoie deux de ses disciples interroger Jésus sur son identité, celui-ci en guise de réponse produit plusieurs guérisons miraculeuses. Sous ces deux scènes de l'Envoi des disciples et des Miracles du Christ, le registre inférieur montre, à gauche, quelques restes architecturés qui laissent croire à un Festin d'Hérode (mais à quel moment? après le retour du chef décollé de Jean ou auparavant, lors de la danse de Salomé ?). Dans la baie à deux formes prennent place, à droite, la Décollation et à gauche, la Remise de la tête par Salomé à Hérodiade. L'histoire s'achève sur la Mise au tombeau du martyr. On peut mentionner aussi la présence du Prodrome, hors de tout cycle, dans la Crucifixion qui figure dans l'axe de l'abside, où il figure comme témoin derrière la Vierge ${ }^{18}$.

16 Guigues Ir, Contumes de Chartreuse, Paris, rééd. 2001, p. 245 (coll. Sources chrétiennes 313). Pour le cas de la chartreuse ducale Sainte-Trinité de Champmol, on se référera au récent colloque Autour du «Puits de Moïse». Pour une nouvelle approche (Dijon, 16-18 octobre 2008), présenté par S. Juge et D. Russo, Autour du "Puits de Moïse». Pour une nouvelle approche, dans Bulletin du centre d'études médiévales d'Auxerre 12/2008, [En ligne] URL: http://cem.revues.org/index7522.html [adresse valide en novembre 2009].

17 Toujours dans la nécessité de restreindre le nombre de clichés publiés, nous renvoyons, pour des vues d'ensemble de ces peintures, au site consacré à la chartreuse, spécialement Visite en images de la chartreuse et Chartreuse numérique [En ligne] URL: http:/www.chartreuse. org/Site/Chartreuse/ChartreuseEnImages/ChartImages.php et http:/www.chartreuse.org/Site/ Chartreuse/3D/ [adresses valides en novembre 2009].

18 Pour C. HECK, op. cit., pp. 435-436, il y a là confirmation d'un choix pontifical, que montrent également la Crucifixion de la chapelle Saint-Martial, dans le palais des Papes, et une troisième dans la livrée d'Aigrefeuille à Avignon, qui comportent toutes trois les deux saints Jean debout de part et d'autre de la croix. A noter que M. LAClotte et D. ThiÉBAut, op. cit., p. 165, contestent l'identification du Baptiste dans la Crucifixion de la chapelle SaintMartial. 


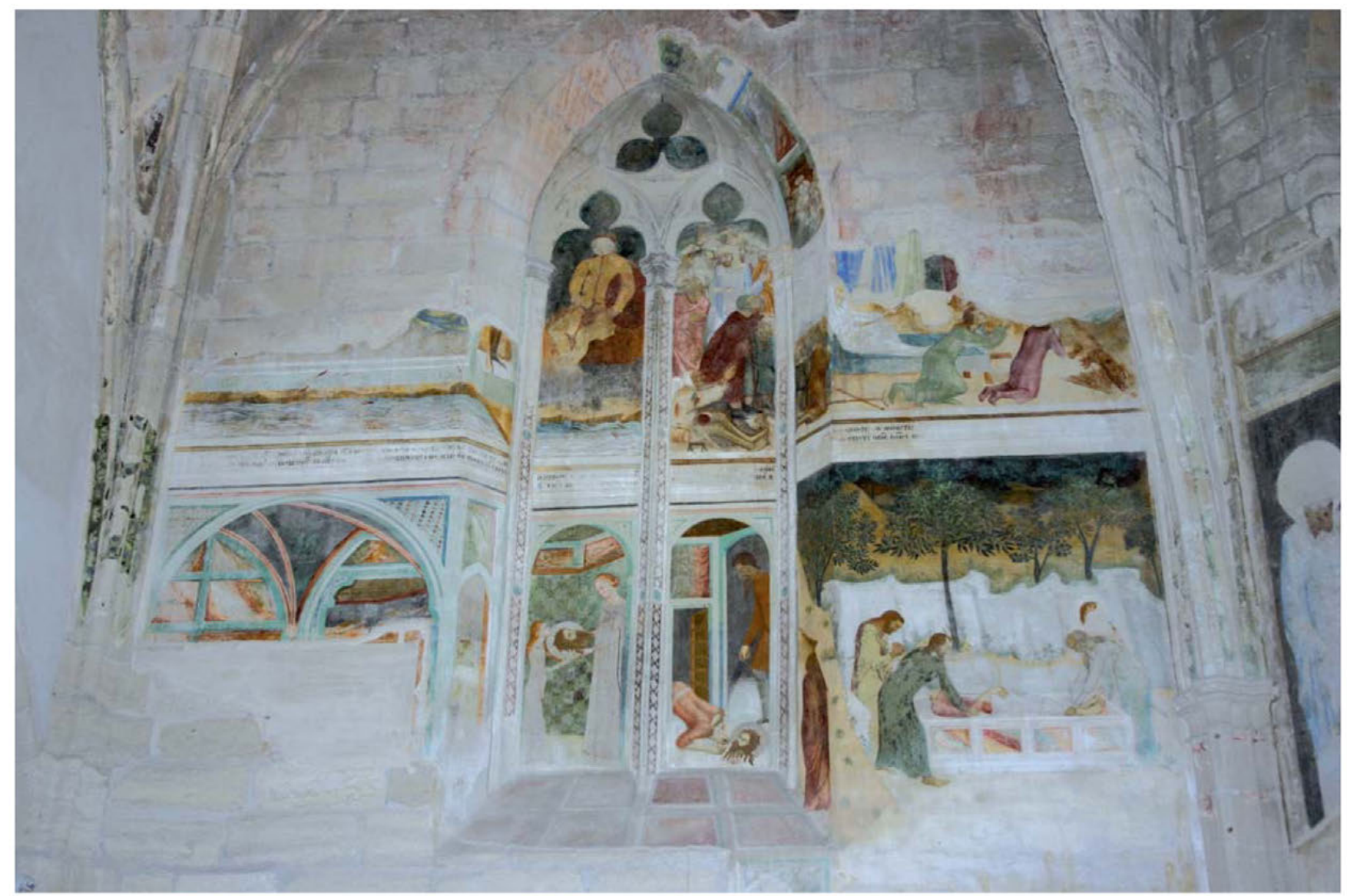

Figure 3 : Villeneuve-lès-Avignon, chartreuse du Val-de-Bénédiction, chapelle du tinel, mur de gauche : registre haut, Envoi des disciples ? (pan gauche), Miracles du Christ (pan droit) ; registre bas, Festin d'Hérode ? (pan gauche), Ensevelissement de Jean (pan droit) 
A travers cette première comparaison d'ensemble, il apparaît qu'au palais pontifical et à la chartreuse, les cycles du Baptiste se déploient sur un espace sensiblement identique (soit sur deux murs découpés en deux registres narratifs). Le décompte des thématiques repérables traditionnellement dans l'iconographique johannique donne même un nombre équivalent de scènes, le cycle du palais étant constitué de onze moments, et celui de la chartreuse également.

\begin{tabular}{|ll|}
\hline \multicolumn{2}{|c|}{ Thématiques comparées des deux cycles de saint Jean } \\
Palais des papes & Chartreuse \\
Annonce à Zacharie & Annonce à Zacharie \\
Visitation & Naissance \\
Naissance & Imposition du nom \\
Imposition du nom & \\
Circoncision & \\
JB et des baptisés & \\
Rencontre avec le Christ (Ecee Agnus) & \\
Dialogue avec les prêtres, lévites et pharisiens \\
Baptême du Christ & \\
Epiphanie du baptême & Envoi des disciples (?) \\
& Guérisons miraculeuses du Christ \\
& Décollation \\
Décollation & Festin d'Hérode (?) \\
Festin d'Hérode et présentation chef & Hérodiade-Salomé-chef JB \\
Salomé-Hérodiade-chef de JB & Mise au tombeau \\
&
\end{tabular}

Le parallélisme strictement formel qui se dégage de cette comparaison ne laisse pourtant pas d'étonner car il faut bien admettre que ce n'est pas l'effet restitué visuellement, ni par le choix des scènes ni par la disposition des images. Y compris dans le détail de la composition de chaque scène, on observe quelques procédés basiques de variation d'un cycle à l'autre, à travers un déplacement thématique ou par la simple interversion de personnages, comme le glissement de l'Imposition du nom vers la scène de la Circoncision, ou, dans la Présentation du chef de Jean, l'échange des places de Salomé et d'Hérodiade (fig. 3). Quant à la Décollation du Baptiste, c'est le décalage de l'instant narratif qui assure un renouvellement plus subtil de la formule, lorsque le temps du martyre, un moment suspendu à Avignon dans l'épée figée sur le col du saint, reprend son cours à Villeneuve où la tête décapitée roule à terre (fig. 4 et 5). Ces quelques comparaisons de détails suffiraient à illustrer les variantes imaginées entre les peintures de 1347 et celles de 1355 , et elles pourraient être prolongées en termes stylistiques, autour de l'intervention évidente de différentes mains sur le chantier. Ainsi Matteo Giovannetti n'a nullement donné à la chartreuse un doublon du palais pontifical. Mieux encore, il nous semble que, tout en puisant dans un formulaire commun, le peintre inscrit le saint dans une mouvance différente selon l'un et l'autre cas. L'examen rapproché du traitement de certains épisodes en confirme la suggestion. 


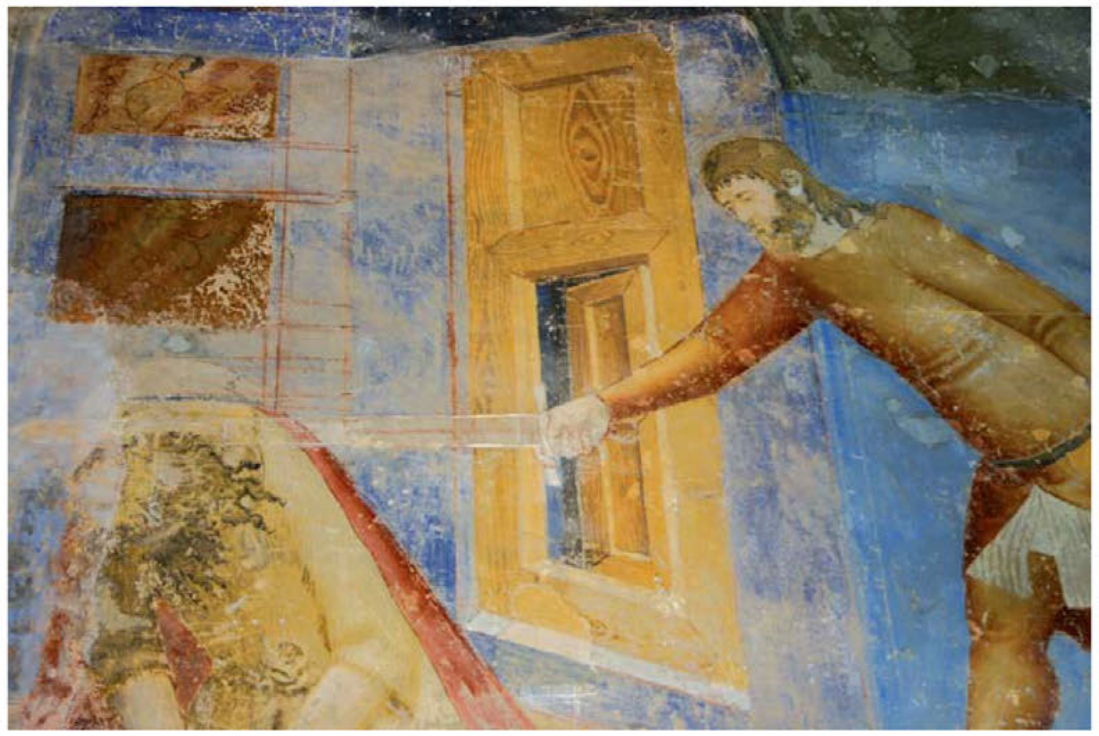

Figure 4 : Avignon, palais des papes, chapelle du consistoire, mur nord, registre bas, cycle de Jean le Baptiste : Décollation de Jean, détail (pan droit)

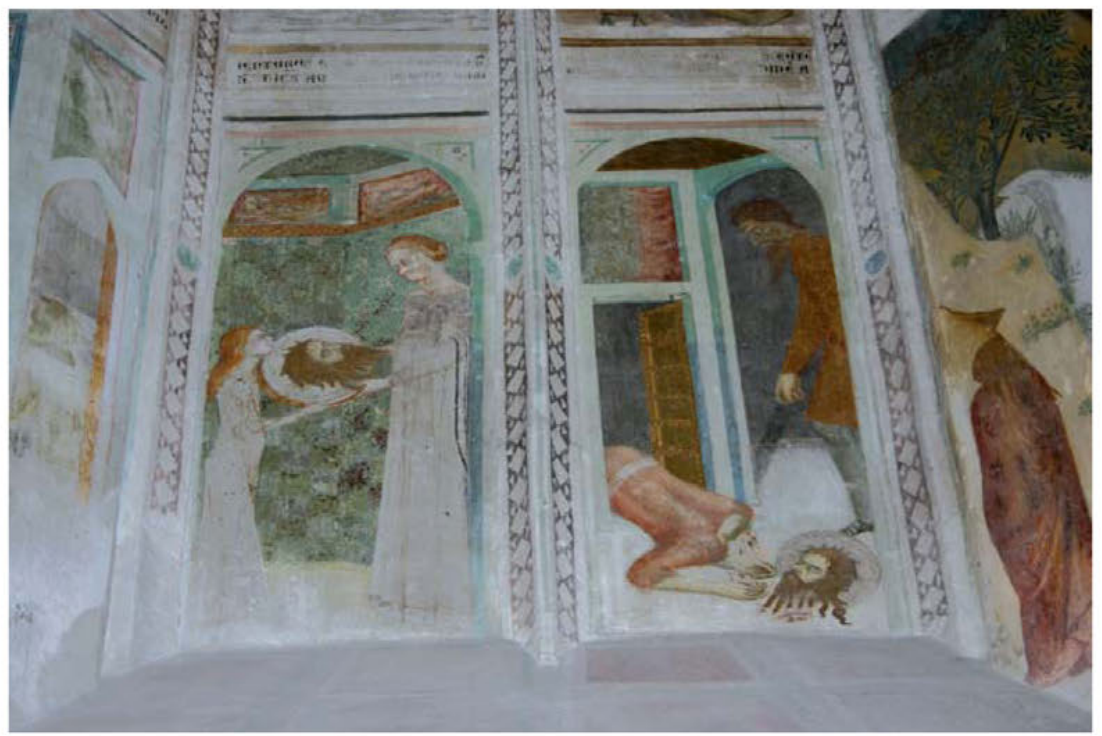

Figure 5 : Villeneuve-lès-Avignon, chartreuse du Val-de-Bénédiction, chapelle du tinel, mur de gauche, registre bas : Décollation et Présentation du chef de Jean (baie aveugle) 
L'Enfance du Baptiste occupe, à la chartreuse, les deux registres superposés du mur de droite, en quatre tableaux et cinq moments ; tandis qu'au palais, elle est limitée au registre supérieur en deux tableaux et trois moments. On notera l'intérêt accordé dans les deux chapelles à l'Annonce à Zacharie, qui place d'emblée le cycle dans l'espace consacré d'un temple, et le motif de l'archange pose la scène en parallèle avec les Annonciations. Dans la chapelle du palais, où un agneau sacrifié rôtit sur l'autel, elle fonctionne également avec la scène souscrite de l'Agnus Dei où le Christ, agneau désigné, est peint à l'aplomb de l'animal du sacrifice. Le texte qui court en bandeau, et qui, à Villeneuve, s'inscrit sur le phylactère de l'ange, assez bien lisible du sol, n'est pas identique ici et là, mais il procède du même passage de l'évangile de Luc (I, 8-13) qui présente la sacralité du lieu et du rituel, et la faveur divine qui touche Zacharie : «Ne crains pas, Zacharie, car ta prière a été entendue et ta femme Elisabeth t'enfantera un fils $\gg{ }^{19}$. Force est de remarquer qu' est oblitérée la citation biblique susceptible d'accompagner aussi la mise en image de l'Annonce à Zacharie, c'est-à-dire les versets qui suivent dans l'évangile (Lc I, 18-22), comportant la menace angélique face à un Zacharie incrédule devant ce miracle tardif, et sa condamnation à la mutité : «Pro eo quod non credidisti verbis meis ${ }^{20}$. Non que la mutité soit négligée dans les deux cycles, puisqu'elle est sousentendue au moment de la Naissance et de la Circoncision par la représentation de Zacharie écrivant le nom du nouveau-né, mais la comparaison avec d'autres choix épigraphiques fait conclure à la volonté de manifester la valeur positive de la parole divine et de l'écriture inspirée.

A la chartreuse, la peinture suit plus littéralement l'ordre du récit de Luc en intégrant la Visitation entre l'Annonce et la Naissance, ce qu'une enquête comparative dans la tradition peinte de Jean donne comme rare, car la Visitation est plutôt ancrée dans les scènes de l'Enfance du Christ. Toutefois, la Légende dorée fait déjà état de l'homélie du Pseudo-Chrysostome, où la Visitation est commentée comme le moment où le Christ, depuis le ventre maternel, confère l'onction prophétique à Jean qui la transmet à sa mère, à travers la salutation d'Elisabeth ${ }^{21}$. Dans la tradition orale des devinettes des moines, une énigme le résume ainsi : «Qui locutus est antequam natus?». La finale de l'inscription de la

19 Détail des inscriptions relatives à 1'Annonce. A Avignon, bandeau inférieur : " (Quomodo Zachar)ie i(n)ce(n)su(m) pone(n)ti appanit Gabriel a(n)g(e)l(u)s dice(n)s Helysabet uxo(r) tua p(ar)iet t(ibi) fliu( $m$ ) et v(ocabis) nomen eius Iohannes [sic]». A Villeneuve, bandeau inférieur : "Quomodo cu(m) sac(er)dotio fungeret(ur), b(ea)t(us) Cacharias in ordine vicis sue (ante) Deum, (secundum) consuetu[dinem sacerdotii, sorte exiit ut] $i(n)$ ce(n) su(m) pon(er) et i(n)gress(us) te(m)plum D(omin)i et om(ni)s multitudo pop(u)li erat ora(n)s foris hora incensi Angelus domini apparuit [illi]"; cartouche de 1'ange : "Ne timeas, Cacharias [sic], quoniam exaudita est deprecatio tua et uxor tua Helisabet pariet tibi filium».

20 Pour un exemple dans l'iconographie italienne des années 1240, au baptistère de Parme, nous nous permettons de renvoyer à notre Saint Jean-Baptiste au baptistère de Parme : naissance d'un cycle biographique, dans Medioevo : Immagine e racconto. Colloque international d'études (Parme, 27-30 sept. 2000), éd. A.C. Quintavalle, Electa, 2004, pp. 349-359.

21 JaCQues de Voragine, op. cit., p. 435, p. 438. 


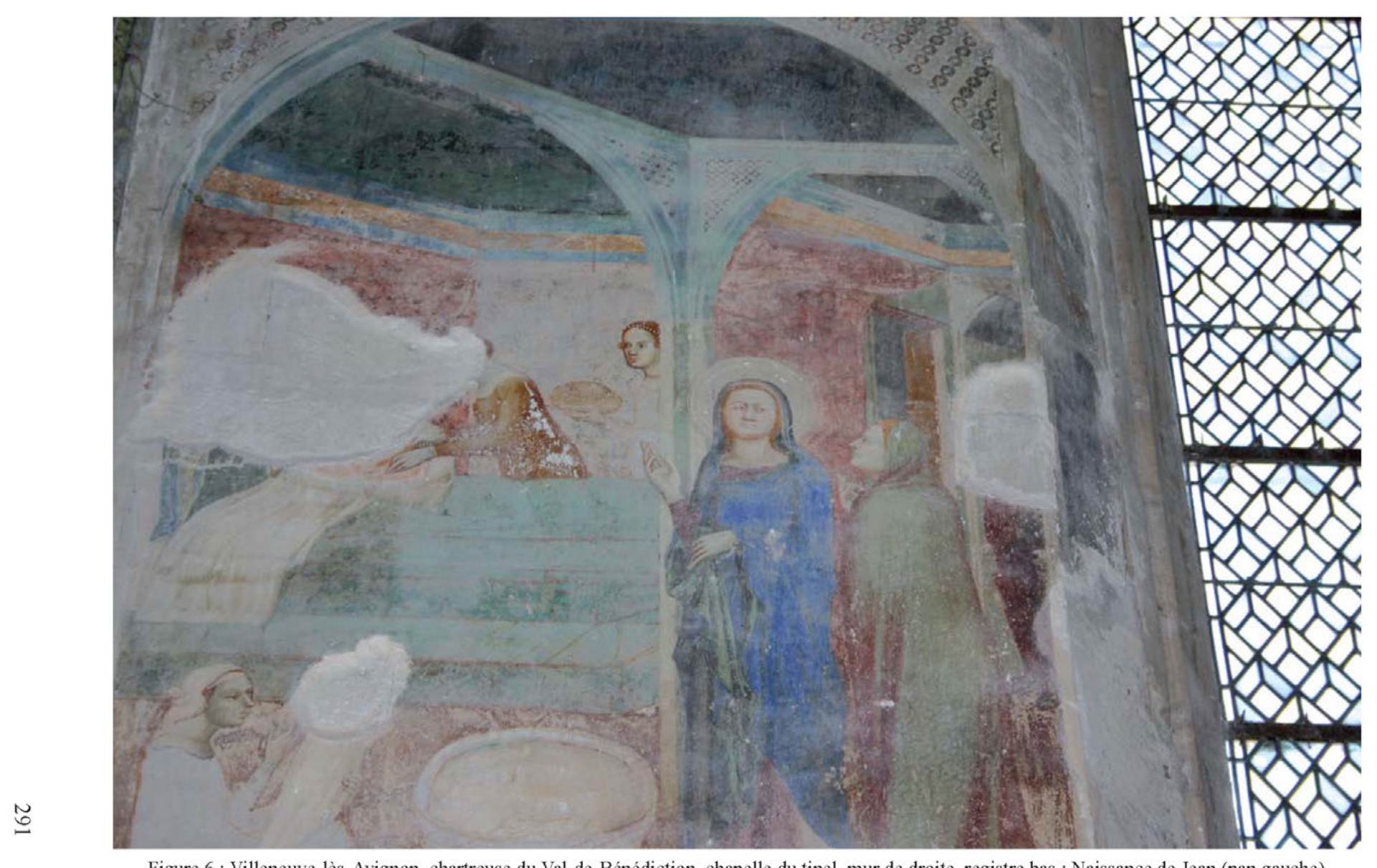

Figure 6 : Villeneuve-lès-Avignon, chartreuse du Val-de-Bénédiction, chapelle du tinel, mur de droite, registre bas : Naissance de Jean (pan gauche) 
fresque pourrait lui servir de solution, par l'extrait de Luc (I, 39-41) : «Exultavit infans in utero ejus». L'ajout à Villeneuve de cette scène, absente d'Avignon, peut éventuellement s'expliquer à travers les joca monachorum, mieux relayés dans le milieu culturel monastique ${ }^{22}$. Autre possibilité, qui me paraît plus probante dans l'univers cartusien, cette Visitation insiste sur la figure mariale, qui est mise en parallèle avec celle du Baptiste, et que les autres scènes viennent souligner ${ }^{23}$. En effet, on retrouve Marie au premier plan de la Naissance de Jean, dont elle montre la scène de la main (une sorte d'Ecce Iohannes Baptista inversé) (fig. 6). Dans la Circoncision adjacente, qui, notons-le, est donnée comme une affaire de femmes, tandis que le groupe des hommes concentre son intérêt sur le nom de l'enfant (fig. 7), le nouveau-né est tenu dans les bras par une femme dans une position privilégiée, mais la tête de ce personnage n'est pas ceinte d'un nimbe, il semble donc impossible qu'il s'agisse encore une fois de la future Theotokos.

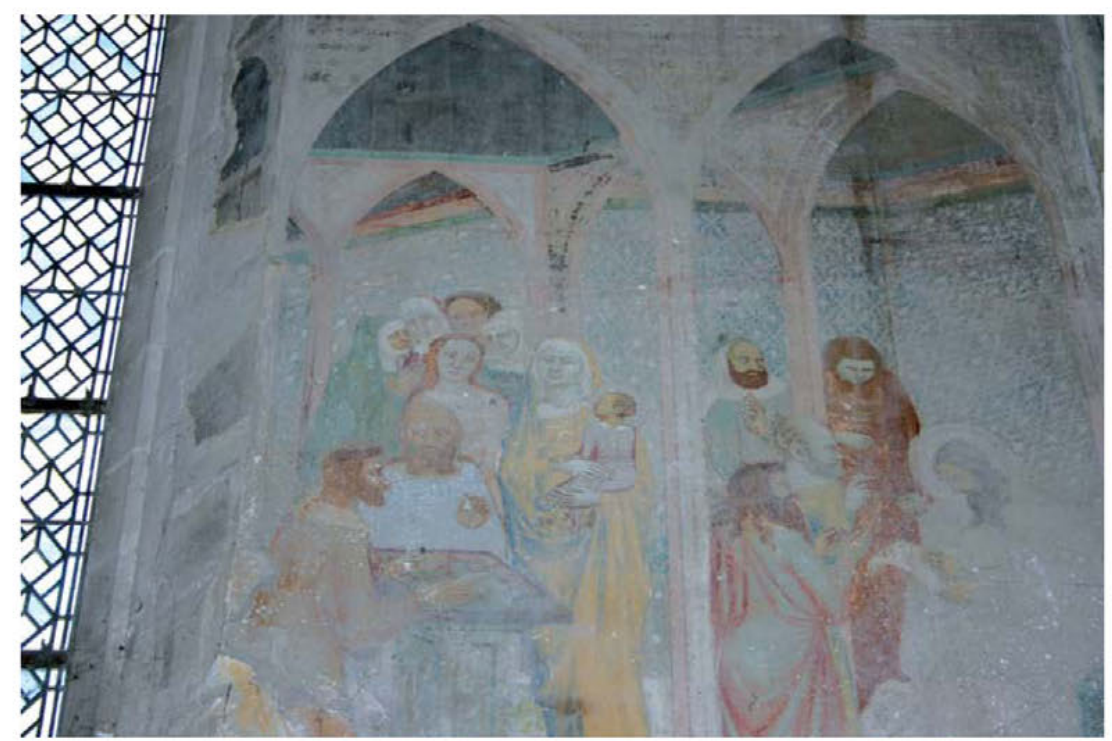

Figure 7 : Villeneuve-lès-Avignon, chartreuse du Val-de-Bénédiction, chapelle du tinel, mur de droite, registre haut : Circoncision et Imposition du nom de Jean (pan droit)

22 Voir B. Roy, Saint Jean-Baptiste et la tradition orale, dans Jean-Baptiste le Précurseur au Moyen Age, Aix-en-Provence, 2002, pp. 178-180 (coll. Senefiance 48)

23 Sur la dévotion mariale chez les chartreux, ef récemment N. NABERT, M.-G. Grossel dir., La figure de Marie en chartreuse /Une dormition de la Vierge, manuscrit inédit de la GrandeChartreuse, Paris, 2009. 
Une hésitation similaire naît au palais des papes, dans la fresque de la Naissance (fig. 8), devant la parente qui porte le nourrisson, mais la même absence de nimbe doit faire renoncer à y voir la Vierge. Stricto sensu, du point de vue des modèles iconographiques, il est évident que le glissement de la formule double « nourrisson au bras d'une femme » à une formule triple «nourrisson au bras d'une femme dont la tête est nimbée» ne transforme pas fondamentalement la composition, mais il influe sur son interprétation. A nouveau, la Légende dorée relaie, en l'attribuant à Pierre Comestor, le récit apocryphe qui accorde à Jean le privilège d'avoir été tenu, dès la naissance, dans les bras de la mère du Seigneur ${ }^{24}$. Il semble bien que la première mise en image du récit soit à dater également de la fin du XIIIe siècle, en Italie ${ }^{25}$. AAvignon, en n'octroyant finalement pas l'attribut de la sainteté à cette parente, malgré sa position fortement valorisée, Giovannetti a pu répondre au désir du commanditaire de ne pas intégrer la légende, pourtant déjà répandue, et de ne rien ajouter à la lettre de l'évangile.

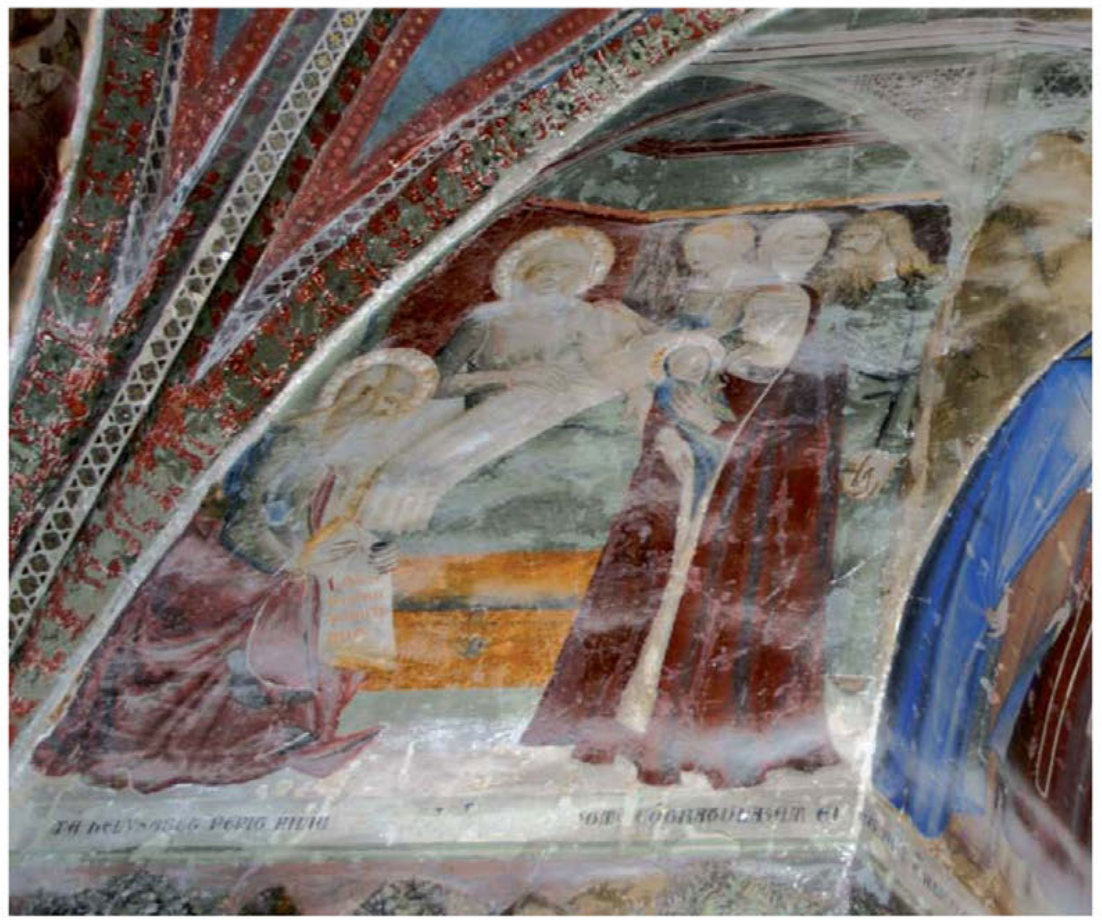

Figure 8: Avignon, palais des papes, chapelle du consistoire, mur est, registre haut, cycle de Jean le Baptiste : Naissance et Imposition du nom de Jean (pan gauche)

24 Jacques de Voragine, op. cit., pp. 434-435 et n. 7, p. 1264.

25 M. Aronberg Lavin, Giovannino Battista: a study in Renaissance religious symbolism, dans The Art Bulletin, vol. 37, 2 (juin 1955), pp. 85-101, spéc. pp. 87-88. 
Dans la chartreuse, en revanche, le lien fort qu'instaurent les peintures entre Marie et Jean (dans deux, voire trois scènes sur les quatre de l'enfance) confirme en écho un aspect de la spiritualité cartusienne, où la Vierge, comme modèle de vie contemplative, est patronne de l'ordre et le Précuseur également à ses côtés, reconnu comme modèle de vie érémitique. Les Coutumes de Guigues signalent d'ailleurs que les premiers ermitages étaient placés sous les vocables conjoints de Notre-Dame et de saint Jean-Baptiste ${ }^{26}$. A Villeneuve, on sait qu'entre la fondation de 1356 et 1362, il s'opère un glissement du vocable même de la chartreuse qui passe de la dédicace au Baptiste au nom de Notre-Dame du Val-de-Bénédiction. Faut-il lire la faveur iconographique accordée ici à Marie comme 1'un des germes de ce changement d'appellation? Du moins, elle confirme que si les fresques sont effectivement antérieures à la fondation, l'expression de thèmes cartusiens, chers à Innocent VI, infléchissait déjà les choix iconographiques.

C'est autour du traitement pictural du ministère du Précurseur que se révèlent les plus importantes divergences entre le cycle du palais des papes et celui de la chartreuse. Le constat est flagrant au décompte même des scènes : au nombre de cinq pour Avignon, au nombre de deux pour la chartreuse - en admettant que la scène détruite représente bien, comme dans les exemples similaires ${ }^{27}$, le saint emprisonné envoyant ses disciples. Il est ensuite confirmé par les choix des scènes. La chartreuse optant pour un Baptiste emprisonné, le récit a finalement déjà basculé du côté de la passion du saint, que l'on suit au registre inférieur. Le cycle villeneuvois paraît alors être focalisé sur les deux extrémités de la vie de Jean, l'enfance au mur droit et le martyre au mur gauche. Et ce d'autant mieux que l'histoire y est menée jusqu'à la Mise au tombeau, alors que les cycles occidentaux en font assez souvent l'économie. C'est d'ailleurs le cas à Avignon, où le récit s'achève ainsi très classiquement sur le Festin d'Hérode au retour de la tête du martyr. Pour autant, d'importants ensembles consacrés à la vie de Jean, entre XIIe et XIVe siècles, comme au baptistère de Florence, à la primatiale de Lyon, ou encore à Müstair ou à Sens, se concluent par l'ensevelissement du saint, et il n'est done pas possible d'y chercher un réseau sous-jacent d'influences iconographiques spécifiques. Quant au choix de montrer la dépouille inhumée sans tête, le sondage que nous avons conduit indique qu'il ne peut être automatiquement rattaché à une tradition précise, puisqu'on le repère dans la première moitié du XIVe siècle tout autant dans la sphère byzantinisante, sur les mosaïques du baptistère de Venise, que dans le royaume de France, à Lyon, sur les ressauts sculptés de la primatiale Saint-Jean ${ }^{28}$.

26 Guigues I ${ }^{\text {er }}$, op. cit., p. 215.

27 Pour d'autres occurrences iconographiques, cf I. HuEck, Das Programm der Kuppelmosaiken im Florentiner Baptisterium, Mondorf-am-Rhein, 1962, pp. 171-172.

28 On trouvera le détail iconographique de ces exemples dans V. Rouchon Moulleron, art. cit. (2007), pp. 43-44. 
Si l'on veut continuer d'examiner la figure du Baptiste élaborée dans la chartreuse, on a ainsi la surprise, pour celui qui est invoqué comme l'ermite par excellence, de ne le trouver jamais représenté au désert. Les décors architecturaux priment dans chaque phase, de l'enfance à la passion, et seule l'ultime scène l'attache, dans la mort, à un paysage rocheux et inhabité. Il est permis de mettre ces images en parallèle avec les nouvelles expériences du désert que développent certaines chartreuses, à partir du XIIIe siècle, loin du modèle initial de la Grande Chartreuse, coupée du monde. Si l'urbanisation galopante est la cause première de l'installation de chartreuses à portée des villes, une autre conception a pu être mise en avant, autour de la notion d'une intériorisation du désert, que le contact avec les Mendiants a peut-être favorisée ${ }^{29}$. Villeneuve est un exemple emblématique d'une chartreuse donnant sur une cité, fondation péri-urbaine, dont le petit désert est alors limité à l'espace de ses cloîtres et des terrains avoisinants. Le Baptiste des peintures de Villeneuve ne vit pas au désert, il y reçoit seulement son tombeau. A sa manière, Innocent VI, en recherchant pour sa sépulture une toute symbolique solitude cartusienne à l'écart de la capitale avignonnaise, n'a-t-il pas emprunté le même parcours que le saint de la fresque?

En outre, à la chartreuse, le moment du Baptême du Christ, pourtant inhérent à l'action de Jean et qui lui confère son surnom de Baptiste, n'est pas même représenté. Ce Précurseur-là ne se montre donc ni ermite, ni baptiseur ${ }^{30}$. Parmi les nombreuses épithètes qui lui reviennent, et qu'énumère la Légende dorée au début du chapitre qui lui est consacré ("prophète, ami de l'Epoux, voix, baptiste du Sauveur », etc.), aucune n'apparaît spécialement valorisée par l'image, si ce n'est, peut-être, l'appellation d' «ange», du fait de la présence d'anges dans les fresques des voûtains de la chapelle. Plusieurs sont effacés, mais il est légitime de considérer que l'ensemble de la voûte en était décoré. Ce nom d'«ange», unanimement attribué à Jean dans la littérature spirituelle latine depuis saint Jérôme, se veut non une définition de sa nature, mais une métaphore de son état, de la pureté de sa vie ${ }^{31}$ — et il représente ainsi un modèle infiniment désirable pour un chartreux.

29 Dans La naissance des chartreuses, op. cit., of les contributions de B. Bligny, Saint Bruno et la naissance des chartreuses, p. 7-14, spécialement p. 12 ; de Dom J. Dubors o.s.B., Le désert cadre de vie des chartreux au Moyen Age, pp. 15-35, spéc. pp. 26-29; et de A. GRARD, Implantation cartusienne..., art. cit., pp. 143-144.

30 Un tel vide iconographique, si exceptionnel dans la série des cycles médiévaux du Baptiste, invite d'ailleurs à s'interroger sur le décor qui aurait pu orner l'arc, actuellement blanchi à la chaux, qui séparait la chapelle du tinel. Nous n'avons pas eu connaissance, au moment de la rédaction de ce travail, de rapports de restaurations le concernant. Il s'agira pourtant d'un point à éclaircir, en particulier dans le cadre des projets de restitution numérique des éléments disparus de la chartreuse (dont les peintures de la chapelle), portés par plusieurs laboratoires du CNRS et le Centre International de Conservation et Restauration du Patrimoine, cf. [En ligne] URL : http:/www.chartreuse.org/Site/Chartreuse/3D/ [valide en novembre 2009].

31 Jaceues de Voragne, op. cit., p. 432. Egalement V. Rouchon Moullleron, art. cit., (2007), p. 41. 
Dans les fresques d'Avignon se dessine un autre profil johannique, riche d'une forte recharge théologique. Le Précuseur s'y décline sous de multiples aspects : celui qui rend témoignage au Christ et baptise, qui fait face aux dignitaires juifs, et qui paye de sa tête d'avoir contesté le roi Hérode, en un mot l'homme de Dieu qui affronte les hommes haut placés, religieux et courtisans. Mention doit être faite ici d'une des fresques qui décorent la voûte de la chapelle, où sont figurés en pied, sur chaque voûtain, le Baptiste et ses parents, et l'Evangéliste et ses parents. Le saint y est présenté indiquant du doigt une hache au pied d'un arbuste, un attribut qui apparemment n'a pas été reconnu dans les restaurations successives, puisque qu'il a été recouvert d'un feston bleu d'encadrement, qui occulte la lame et ne laisse plus voir, aujourd'hui, que le manche de bois (fig. 9) ${ }^{32}$. Et le phylactère que tient Jean évoque la cognée déjà positionnée à la racine de l'arbre, prête à couper celui qui ne produit pas de bon fruit : «Iam securi(s) ad radicem arbo( $r) i(s)$ posita est» (Mt 3, 10). Dans ce type d'image non narrative, qui fige les portraits hagiographiques, au physique comme au spirituel, la citation retenue n'a done pas été l'Ecce Agnus Dei, également classique dans les cartouches attribués au saint, où l'insistance porte sur un Baptiste annonciateur du Christ. A travers le choix de ces paroles comminatoires du Prodrome, le rouleau écrit se fait l'écho de ses appels au repentir et à la pénitence, c'est-à-dire à l'une des formes essentielles de sa charge pastorale.

E. Castelnuovo a relevé la profusion des écritures exposées dans la chapelle, qui ponctuent et animent le récit peint. Il les a rapportées à la description du décor perdu du consistoire voisin, citée par Baluze dans sa vie de Clément VI, dans lequel peinture et écriture se développaient en parallèle autour de thématiques liées au jugement, au droit et à la justice. Dans cette perspective, censée se prolonger dans la chapelle adjacente, il propose d'expliquer la présence conjointe des deux Jean comme «l'un le précurseur et l'autre l'exégète et le codificateur de la nouvelle loi $)^{33}$.

Avec l'inflexion spéciale que montrent le phylactère du portrait en pied peint à la voûte, ainsi que les inscriptions des autres murs, l'aceent interprétatif me paraît devoir porter surtout sur la parole. Ainsi, pour l'épisode du dialogue entre Jean et les pharisiens rapporté dans le quatrième évangile ( Jn 1, 19-26), on observe une mise en scène picturale et épigraphique d'une grande habileté, à travers les deux cartouches déroulés en parallèle dans la main du Baptiste et celle de l'autre protagoniste. Sous le feu des questions posées ( $T u q(u i) s$ es, Helya(s) es tu, $P(r)$ oph(et)a es tu, Qui(d) (er)go dicis de te ipso, Quid (er)go baptiças si tu no(n) es

32 En réintégrant cette hache qui, à ma connaissance, n'a jamais été signalée, on restituera en premier lieu au geste du doigt du saint la monstration de cet objet (dont les occurrences sont nombreuses par ailleurs), et non une allusion, proposée par M. LACLOTTE et D. Thí́BAUt, op. cit., p. 172, au bois de la croix de la Crucifixion peinte sur le mur voisin.

33 E. Castelnuovo, op. cit., pp.104-107 (1991), citation trad. p. 118 (1996). 


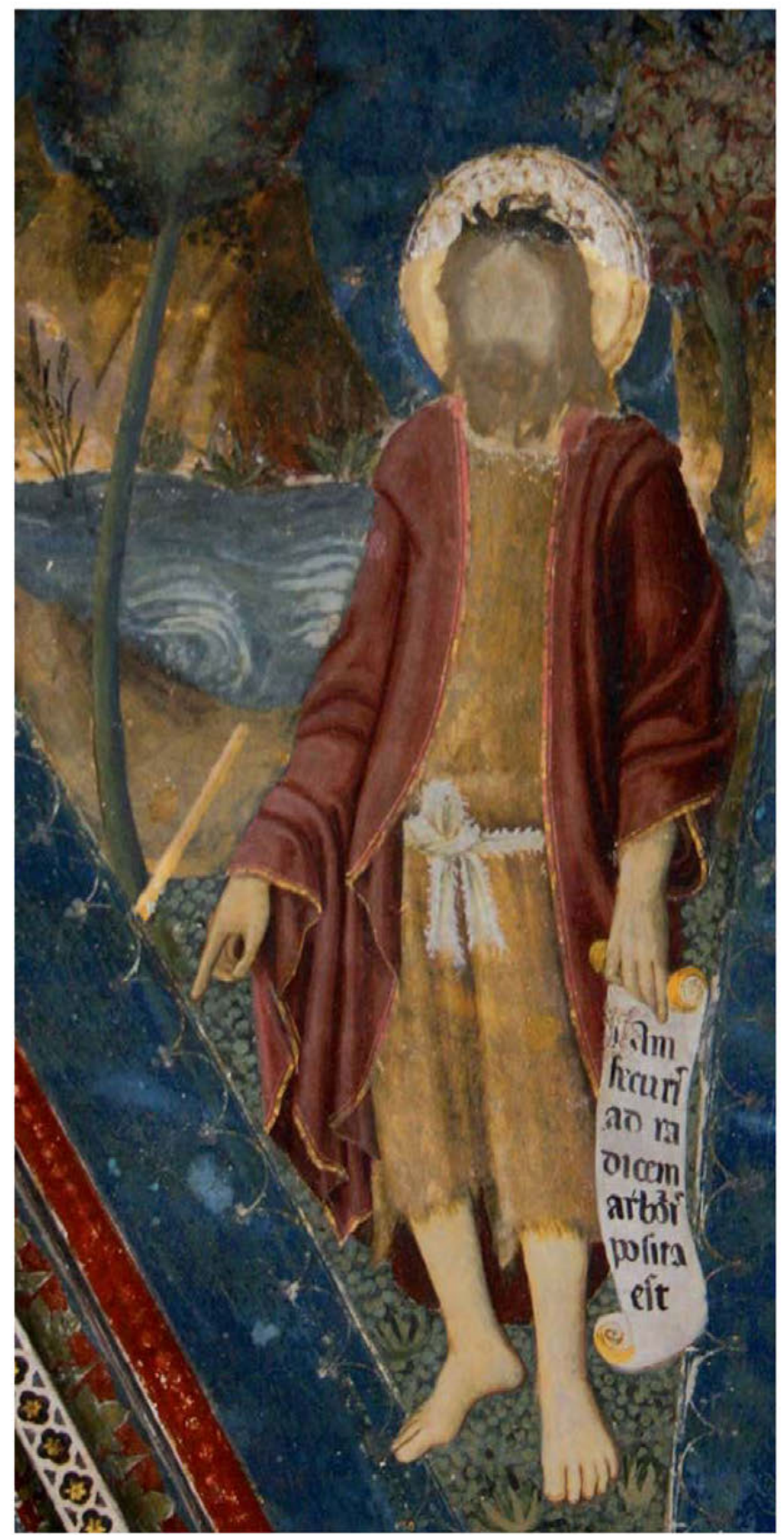

Figure 9: Avignon, palais des papes, chapelle du consistoire, voûtain nord : Jean en pied indiquant la hache à la racine de l'arbre 
propheta, Helyas nec [...]»), Jean répond : «No(n) su(m) ego XP(istu)S, non sum, non, Ego vox clama(n)tis i(n) deserto, Pa(ra)te viam Do(mi)ni, Ego baptiço in aqua» (fig. 10). Il se définit donc positivement comme étant une vox. Et dans le bandeau inscrit sous la scène du baptême, le titulus latin commence par «Comment le verbe du Seigneur a été donné au bienheureux Jean de Zacharie » ${ }^{34}$, avec cette fois l'emploi du terme verbum, que l'on retrouve également en exergue sur la voûte, dans le voûtain voisin de Jean l'évangéliste, qui exhibe un phylactère où se donne à lire le début de son évangile (In pri(n) cipio erat verbu(m) 7 [=et] verbu(m) erat ap(ud) Deu(m) et Deus erat verbu(m), Hoc erat i(n) pri(n)c[...], Jn 1, 1-2).

Il me semble donc que, par ce jeu de positionnements et d'inscriptions, le décor dessine spécifiquement un profil du saint en tant que détenteur de la parole, et qu'il propose ce profil au collège cardinalice régulièrement réuni dans le consistoire voisin. En complémentarité du modèle binaire «écriture-peinture» qui a pu être discerné dans le projet décoratif conçu pour la salle du consistoire, la pensée iconographique mise en œuvre dans la chapelle paraît instaurer un troisième terme essentiel livré au regard de ces maîtres de la parole qui fréquentaient les lieux, dans une triade «scriptura-pictura-verba). La même suggestion naît pour Jean l'évangéliste. Car, sur les pans de mur opposés, l'auteur du quatrième évangile et de l'Apocalypse n'est jamais fixé dans l'attitude, pourtant inhérente à sa fonction scripturaire, de l'écrivain. A Patmos, il n'est pas figuré en scribe, mais à genoux devant le Fils de l'homme dont la bouche est transpercée du glaive de la parole tranchante $^{35}$. Le pouvoir de parole du Baptiste, tel que le définissent dans cette chapelle les choix picturaux et textuels, se caractérise moins comme modèle d'une prédication efficace que par son effacement au profit de la parole de Dieu - la voix préparant la voie. Ses répliques aux pharisiens sont en effet construites sur la dénégation, où les tournures Non sum, non..., apparaissent répétées sur les quatre lignes initiales. Car la réponse ultime à leurs questions est donnée au sommet de la fresque par les cieux ouverts où le buste de Dieu le Père désigne Jésus comme son Fils. Ainsi, dans ces peintures, Jean est montré non pour ses talents de prédicateur, mais comme voix prophétique qui porte le message divin, et qui, munie de cette mission, ne faiblit ni ne tremble devant les puissants. Il correspond bien, par cette ambivalence, à la figure que façonnaient de lui les prédicateurs à Paris à la fin du siècle précédent ${ }^{36}$.

34 «Q(u)om(od)o f(a)c(tu)m e(st) u(er)bu(m) D(omi)ni s(upe)r b(ea)t(u)m Ioh(ann)e(m) Çacharie $[. .]$.

35 Pour un échantillon comparatif, voir R. M. Dessi, Images médiévales d'une île sainte: Patmos, dans Lérins, une île sainte dans l'Occident médiéval, éd. Y. CoDou - M. Lauwers, Turnhout, 2010 (Collection d'études médiévales de Nice, vol. 9), pp. 213-259. Nous nous proposons de revenir ailleurs sur une lecture d'ensemble de l'histoire de l'évangéliste dans la chapelle pontificale.

36 N. Bériou, L'avènement des mâtres de la Parole. La prédication à Paris au XIIIe siècle, Paris, 1998 (coll. des Etudes augustiniennes, 32), vol. I, pp. 37, 264, 306-307. 


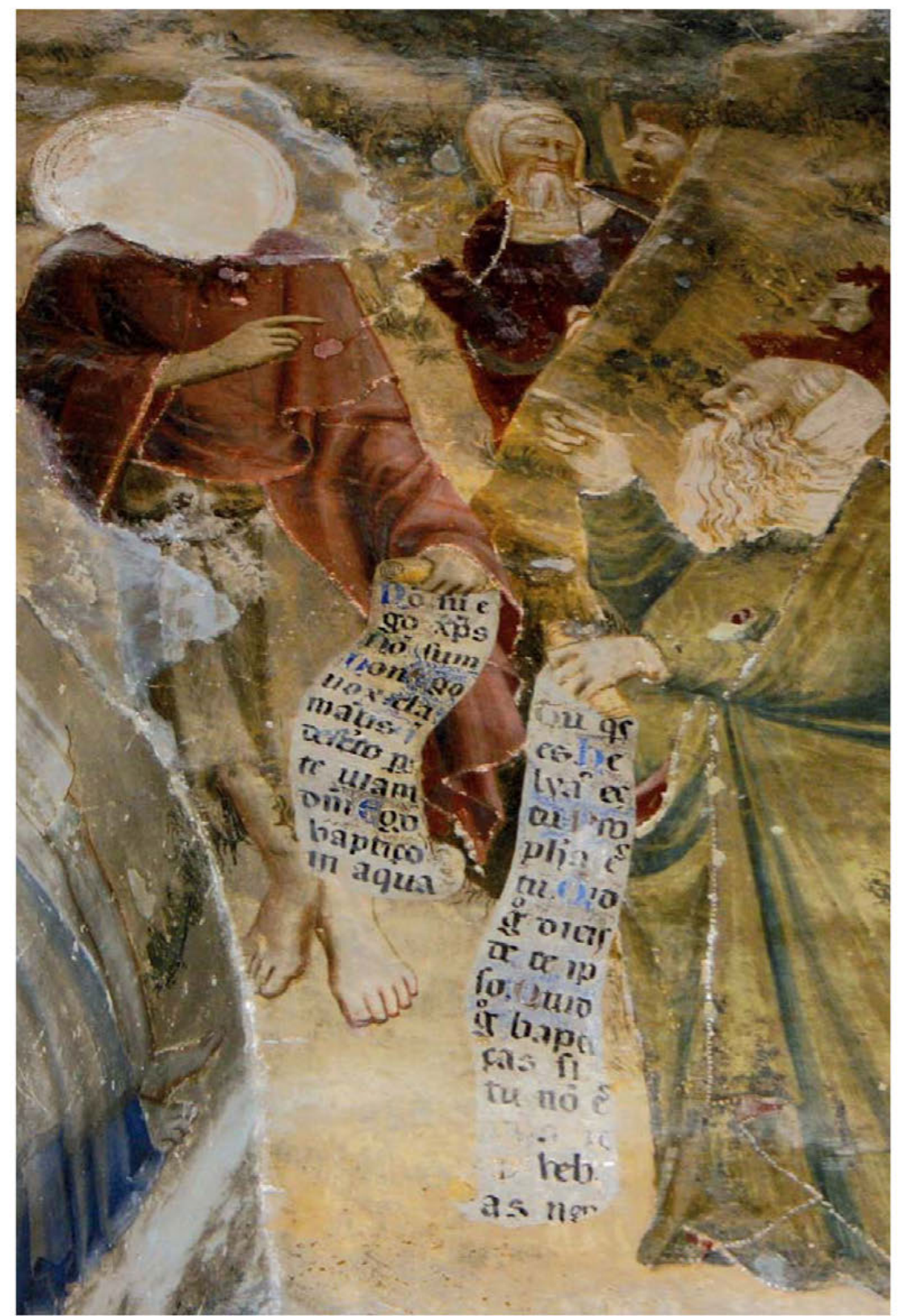

Figure 10 : Avignon, palais des papes, chapelle du consistoire, mur nord, cycle de Jean le Baptiste: Jean interrogé par les pharisiens (détail des phylactères) 
Une telle préminence accordée à la parole, à la bouche, à la voix, pour un décor destiné à la chapelle du consistoire, ne renvoie-t-elle pas, par une concordance interprétative, à la fonction pressentie des cardinaux réunis en ces lieux ? Elle appelle en particulier un rapprochement avec le cérémonial de la promotion cardinalice, spécialement dans son ultime étape dite de l'ouverture de bouche, l'aperitio oris, que le pape prononce sur le nouveau cardinal en ces termes: «Nous t'ouvrons la bouche, ou vous ouvrons la bouche, s'ils sont plusieurs, tant pour prononcer des discours que pour donner des conseils, pour élire le pontife romain et pour tous les actes qui, dans le consistoire ou bien en dehors, sont de la compétence des cardinaux et qu'ils ont coutume d'accomplir ${ }^{37}$.

Dans le cérémonial de Jacques Stefaneschi, qui prolonge celui du XIIIe siècle en introduisant des compléments issus des premiers pontificats d'Avignon, la création des nouveaux cardinaux est décrite sur quatre jours (le mercredi des Quatre-temps, puis les vendredi, samedi et dimanche consécutifs) et s'achève, en une cinquième phase, qui se déroule au cours du deuxième (ou troisième) consistoire suivant, avec ce rite de la bouche ouverte, ainsi que la remise des titres et des anneaux ${ }^{38}$. Au jour du samedi, particulièrement dense, le pontife rassemble dans la salle consistoriale les cardinaux déjà créés et les consultent, tandis que les nouveaux, d'abord maintenus dans une pièce à part dont l'emplacement n'est pas donné, viennent ensuite convoqués et introduits un à un. Mais ce jour-là, leur bouche est encore close. Car ce n'est qu'au cours du consistoire suivant (ou lors de celui d'après), que les nouveaux cardinaux, d'abord mis à l'écart, tandis que les anciens ont encore été consultés sur l'ouverture éventuelle de leur bouche, puis appelés à l'intérieur de la salle, se voient concéder le droit à la parole cardinalice. Principal théâtre des aspects collectifs de cette cérémonie, la salle du consistoire fait ainsi l'objet d'une recharge de sa signification fonctionnelle et spatiale, par le biais d'une chorégraphie bien réglée, qui met en scène l'exclusion et l'intégration entre ses murs des récents promus. La description de la journée du samedi montre, en outre, un savant ballet, par lequel les anciens cardinaux, deux par deux, font pénétrer chaque nouveau dans le consistoire, et elle indique que leur circuit passe devant un autel, dont il est précisé qu'il doit être alors préparé dans la salle où le seigneur pape tient le consistoire ${ }^{39}$. Sans que l'on puisse affirmer, dans la topographie du palais, l'identification de cet autel avec celui de la chapelle adjacente, néanmoins plusieurs aspects de cette cérémonie et la fonction même du consistoire trouvent des échos dans les choix

37 «Nos aperimus tibi os, vel vobis, si sint plures, tam in collationibus quam in consiliis, et in electione Romani pontificis, et in omnibus actibus tam in consistorio quam extra, qui ad cardinales spectant et quos sunt soliti exercere», dans M. Dykmans éd., Le cérémonial papal de la fin du Moyen Age à la Renaissance, t. II, De Rome en Avignon ou le cérémonial de Jacques Stefaneschi, Bruxelles-Rome, 1981 (Bibliothèque de 1'Institut historique belge de Rome, 25), p. 484, §39. Mes remerciements sincères vont à Pierre Jugie pour cette précieuse suggestion.

38 M. Dykmans éd., t. II, op. cit., p. 475-485.

39 M. Dykmans éd., t. II, op. cit., p. 479, § 19-21. 
décoratifs de la chapelle, autour du rite de la bouche ouverte et de la bouche close, ou encore dans la mise en scène binaire des cardinaux appariés. Plus largement, l'épineux problème de la multiplicité des voix dans une même assemblée, crucial devant la pluralité des cardinaux réunis dans le consistoire, se résout dans le couple des saints Jean, semblables par leur nom, différents par leur usage de la voix et du verbe, mais réunis, à travers leur dualité, dans une même parole inspirée.

Dans les remarques qui précèdent, on a cherché à montrer comment, entre Avignon et Villeneuve, Matteo Giovannetti et les concepteurs des fresques ont puisé dans le répertoire iconographique et épigraphique qui leur permettait d'acclimater la figure du Précurseur à des destinations différentes. Les qualités d'adaptations doctrinales et iconographiques, que le Baptiste avait déjà révélées auprès des Mendiants au cours du siècle précédent, lui confèrent des usages protéiformes dont peuvent se targuer bien peu de saints ${ }^{40}$. Jean à la curie est proposé comme l'exemple d'un ministère pastoral, audacieux et prophétique, au milieu des cleres et face aux puissants de ce monde. Mais dans la chartreuse il est dénué de toute charge pastorale, il grandit dans un univers clos et sacré, vit en prison et est inhumé au désert, à l'écart du monde.

Derrière cette étude comparée du Baptiste curial et du Baptiste cartusien, viennent poindre des notions de copie et de transfert. Ces notions, analysées pour les périodes hautes ou centrales, sont rarement étudiées dans les mêmes termes pour le Moyen Age tardif et moins encore pour la peinture murale ${ }^{41}$. Pourtant, tout en délaissant les grands modèles de l'Antiquité ou du premier christianisme, il semble qu'entre Villeneuve et Avignon fonctionne là également le principe de la citation partielle et incomplète, dont l'usage a été démontré depuis les travaux de Richard Krautheimer dans ce qu'il nommait une iconographie de l'architecture médiévale ${ }^{42}$. De même, pour résumer la pensée de Krautheimer, que toute église de l'Occident médiéval pouvait être revendiquée comme une copie du Saint-Sépulcre de Jérusalem, sur une base extrêmement limitée d'éléments communs ou jugés similaires, de la même façon, le modèle de la chapelle avignonnaise commandée sous Clément VI apparaît comme sous-jacent à Villeneuve, mais au titre seulement d'un principe d'autorité, issu d'un prestigieux prototype, car la conception iconographique de la chartreuse est, à l'évidence, radicalement différente de celle du palais. Mieux encore, on a parfois l'impression que Matteo Giovannetti, pourtant au faîte de sa réputation en 1355, s'est appliqué, y compris dans les scènes communes

40 V. Rouchon Moullleron, art. cit. (2007), pp. 40-42.

41 Voir, par exemple, Ideologie e pratiche del reimpiego nell'alto medioevo, Atti della settimana di studio (Spolète, 16-21 avril 1998), 2 vol., Spolète 1999 (Centro italiano di studi sull'alto medioevo, 46) et récemment P. TOuBert, P. Moret éds., Remploi, citation, plagiat, Conduites et pratiques médiévales (Xe-XIle siècle), Madrid, 2009 (Collection de la Casa de Velázquez, 112).

42 R. Krautheimer, Introduction à une «iconographie de l'architecture médiévale» (1942), A. Girod trad., Paris, 1993 (coll. Emblemata). 
aux deux cycles, à accorder un traitement de détail différent à chaque élément, procédant non par auto-citation, mais par une sorte d'anti-citation, perceptible seulement pour qui connaît, en amont, le modèle de l'auctoritas. Assurément, de tels choix iconographiques et épigraphiques ont été opérés pour que la chartreuse abrite non pas un deuxième cycle de Jean, mais un cycle tout autre, à la fois riche du modèle curial et totalement nouveau et autonome. 